\title{
Antitumoral activity of curcumin: an adjuvant therapeutic strategy
}

\author{
Giovanni Tomasello, ${ }^{1}$ Marco Giammanco, ${ }^{2}$ Margherita Mazzola, ${ }^{1}$ Provvidenza Damiani, ${ }^{3}$ William Ra', \\ Paola Giammanco, ${ }^{4}$ Francesca Rappa, ${ }^{1}$ Alessandro Pitruzzella, ${ }^{1}$ Dario Saguto, ${ }^{1}$ Benedetto Di Trapani, ${ }^{5}$ \\ Giovanni Taverna, ${ }^{4}$ Mike Carini, ${ }^{6}$ Antonio Ciulla, ${ }^{5}$ Francesco Carini ${ }^{1}$ \\ ${ }^{1}$ Department of Biomedical Neuroscience and Advanced Diagnosis; ${ }^{2}$ Department of Surgical Oncological and Oral \\ Sciences, University of Palermo; ${ }^{3}$ University Hospital "P. Giaccone", Palermo; ${ }^{4}$ School of Medicine and Surgery, \\ University of Palermo; ${ }^{5}$ Department of General Surgery, Torina Hospital, Palermo; ${ }^{6}$ University of Milano, Italy
}

\begin{abstract}
Curcumin, an active substance contained in an Indian spice called turmeric or curcuma, is well known for its anti-inflammatory and antioxidant properties. In recent times, it was taken into account and studied as an antitumoral molecule, by relying on its interference on several biological mechanisms, such as the inhibition of inflammatory mediators, the enhancement of detoxifying enzymes' action and processes of cell growth and proliferation. Studies show how the high dosage of curcumin in vitro inhibits colorectal tumor cells, pancreatic tumor cells, lung cancer cells and glioblastoma's cells, interfering in signaling pathways as Wnt/ $\beta$-catenin, NF-kB and $\mathrm{PI} 3 \mathrm{~K} /$ Akt and leading to cell cycle arrest and apoptosis. Furthermore, the association between curcumin and chemotherapeutic agents such as 5-fluorouracil strengthens the drug's cytotoxicity and cells' susceptibility towards the chemotherapeutic agent.
\end{abstract}

\section{Introduction}

Curcumin is a bioactive yellow pigment, whose chemical name is 1,7-bis(4-hydroxy-3-methoxyphenyl) hepta-1,6-diene3,5-dione and whose formula is $\mathrm{C} 21 \mathrm{H} 20 \mathrm{O} 6 .^{1}$ \footnotetext{
del Vespro, 129 - 90127 Palermo, Italy.

E-mail: giovanni.tomasello@unipa.it stomach cancer; lung cancer; therapeutic adjuvant.

Received for publication: 27 April 2019.

Accepted for publication: 28 November 2019.

${ }^{\circ}$ Copyright: the Author(s), 2020

Licensee PAGEPress, Italy

Journal of Biological Research 2020; 93:8252

doi:10.4081/jbr.2020.8252
}

Correspondence: Giovanni Tomasello, Department of Biomedical Neuroscience and Advanced Diagnosis, University of Palermo, Via

Key words: Curcumin; colon cancer; chemoprevention; antitumor activity; 5-fluorouracil; nutrition; glioblastoma; pancreatic cancer;

Conflict of interest: the authors declare no potential conflict of interests.

This article is distributed under the terms of the Creative Commons Attribution Noncommercial License (by-nc 4.0) which permits any noncommercial use, distribution, and reproduction in any medium, provided the original author(s) and source are credited.
It is contained in turmeric, an Indian spice that can be obtained from the root of the plant Curcuma longa, together with other two curcuminoids, desmetossigurcumin (20-27\%) and bisdesmetossigurcumin (10-15\%). Curcumin represents the $60-70 \%$ of curcuminoids available in a rough extract of the root. ${ }^{2}$

According to several studies, curcumin is considered as a highly anti-inflammatory and anti-microbial substance, and lately, it is being studied for its antitumoral effects. , $^{3,4}$

Experiments made on murine models show how curcumin plays a preventive role in induction and progression of carcinogenesis, and therefore a chemopreventive role has been profiled for the substance.

Curcumin takes part in carcinogenetic mechanisms thanks to its ability in affecting cell proliferation, cell cycle (especially G2/M arrest), apoptosis, protein folding, proteolysis and translation processes, ${ }^{5}$ and it's mainly involved in the following patterns: i) Antioxidant effect related to the up-regulation of some detoxifying enzymes, such as glutathione S-transferase; 2,4 ii) Inhibition of the expression of some substances that are involved in the inflammation process, such as cyclooxygenase 2 (COX-2), prostaglandins, lipoxygenases (LOX), inducible nitric oxide synthases (iNOS), interferon gamma (IFN $\gamma)$, tumor necrosis factor alpha (TNF $\alpha)$, and interleukins $1,2,6,8$ (IL-1, IL-2, IL6, IL-8). ${ }^{2,4,6}$ The previously mentioned inhibitory effect is being enabled by an adjustment mechanism towards Toll-Like Receptors (TLR), which are responsible for both inflammatory and innate immunity processes. The TLR involved are TLR4 (activating Nf-kb pathway and innate immunity), TLR2 (activating innate immunity), and TLR9 (located into intracellular vesicles of B-lymphocytes, monocytes and natural killer cells, activated by bacterial and viral DNA and initiator of proinflammatory responses that lead to cytokine production; ${ }^{6}$ iii) Inhibition of molecules, such as metalloproteinase 9, involved in the metastatic proliferation of tumors, ${ }^{7}$ iv) Inhibition of $\mathrm{kB}$ nuclear factor, responsible for both carcinogenesis induction and proliferation, and in the development of resistance towards anti-cancer therapies; ${ }^{2,7}$ v) Apoptotic effect on therapies-resistant tumoral cells, related to telomerase enzyme down-regulation; ${ }^{7}$ vi) Interference towards PI3K/Akt signaling pathway, relevant for growth factors in vivo, that can activate the anti-apoptotic mechanism and promote glucose and proteins synthesis, in order to stimulate cells growth and proliferation. This signaling pathway is unusual, as in tumoral cells case, and is able to lead to an abnormal increase of cells growth, proliferation, metabolism, and anti-apoptotic effects, involved in the development of the majority of cancers; ${ }^{8}$ vii) Anti-apoptotic effect related to the intracellular activation of redox reactions, that leads to reactive oxygen species (ROS) production, thereby causing an up-regulation of apoptosis receptors on tumoral cells' membrane. ${ }^{9}$ 
Taking note of the numerous mechanisms in which curcumin intervenes, we propose to investigate in more detail, in the following paragraphs, the role of the above mentioned molecule in specific tumoral pathologies. The tumor cells that, according to the data in the articles examined, are affected by the action of curcumin, have been observed in cancer pathologies like colorectal cancer, pancreatic cancer, stomach cancer, lung cancer and glioblastoma. Positive results were also found in laryngeal, breast, prostate and liver tumors. The aim of the article is therefore to underline the importance of the action of curcumin, to stimulate scientific investigations and to investigate the possible role on the same pathologies about human organisms in order to take into consideration curcumin in the numerous therapeutic protocols of the above mentioned diseases.

\section{Materials and Methods}

In the elaboration of the article about 30 scientific articles were analyzed using the PubMed search engine. The key words used were: curcumin, colon cancer, chemoprevention, antitumor activity, 5-fluorouracil, nutrition, glioblastoma, pancreatic cancer, stomach cancer, lung cancer, therapeutic adjuvant. Of the numerous articles resulting from the insertion of the keywords, only those about biochemical mechanisms of oncological pathologies or strategic therapeutic activities have been examined, starting from the established anti-inflammatory activity of curcumin.

\section{Colon-rectum}

Colorectal cancer is one of the most common cancer in industrialized countries and one of the most relevant causes of death for tumoral pathologies. ${ }^{10,11}$

It's a multifactorial pathology that includes, among its causes, a chronical inflammatory status. This can be related to intestinal dysbiosis, ${ }^{12}$ oxidative stress, ${ }^{11}$ and chronic Inflammatory Bowel Diseases (IBD), such as Morbus Crohn, ulcerative colitis, and nonspecific colitis. ${ }^{13-15}$

Several studies have been performed in animal models, in order to test the effect of curcumin on colorectal tumoral cells.

It was highlighted that curcumin, administrated to APC/Min+ mice, reduces the neoplastic progression of intestinal polyps, enhancing DNA repairing processes, increasing mutated cells apoptosis and reducing colic inflammatory processes. ${ }^{16}$

In Min mice, affected by Familial adenomatous polyposis, curcumin has shown to be effective in lowering the $64 \%$ of colorectal tumor formation. ${ }^{4}$

Apart from being active by itself, curcumin is capable of a synergic action with other active substances. For instance, when combined with silibinin, reduces polyps' formation decreasing inflammatory processes, inhibiting angiogenesis, lowering $\beta$-catenin levels and inhibiting colorectal stem cells proliferation. ${ }^{16}$

When in conjunction with quercetin, curcumin comes up to be effective in adenomatous polyps' qualitative and quantitative reduction. This effect was reported in patients with FAP, previously surgically treated with colectomy. ${ }^{4}$

Curcumin's antitumoral activity towards colorectal cancer is due to the suppression of $\mathrm{Wnt} / \mathrm{\beta}$-catenin signaling pathway (towards down-regulation of $ß$-catenin) and the expression of some microRNA (miR), mainly miR130. These signaling pathways are involved in tumoral proliferation, and for this reason, it appears that curcumin favors a repressive mechanism of action on signaling pathways than on apoptosis induction. ${ }^{17}$

\section{Pancreas}

Curcumin has shown antitumoral effects even on pancreatic cancer's cell lines (PCa, PANC1, and BxPC3). The administration of curcumin doses of $40 \mu \mathrm{g} / \mathrm{mL}$ to PCa cells, $80 \mu \mathrm{g} / \mathrm{ml}$ to PANC1 cells and $10 \mu \mathrm{g} / \mathrm{mL}$ to BxPC3 cells has shown a cell cycle arrest in $\mathrm{G} 2 / \mathrm{M}$ phase in all three the cell lines. It has also been shown that the administration of $80 \mu \mathrm{g} / \mathrm{mL}$ of curcumin on PANC1 cells and of 10 $\mu \mathrm{g} / \mathrm{mL}$ on BxPC3 cells causes both an enhancement of apoptosis rate and of the expression of the anti-apoptotic molecule Bax, together with the downregulation of the anti-apoptotic molecule BCL-2. ${ }^{1}$

In PCa cells, curcumin down-regulates the expression of mTOR protein and induces the expression of LC3II protein, furthering in this way the creation of autophagosomes in PCa cells and the consequential autophagy. ${ }^{1}$

It is shown that curcumin, together with gemcitabine, a drug used for pancreatic cancer's treatment, enhances the rate of cell cycle arrest in the $\mathrm{G} 2 / \mathrm{M}$ phase, significantly increasing apoptosis rate. 1

Lastly, a phase 2 trial on patients with advanced pancreatic carcinoma shows that the daily oral administration of $8 \mathrm{~g}$ curcumin had reduced the tumor by $73 \%$ in one of the patients treated, although it improved neither prognosis nor the survival, suggesting that the patient could have had a curcumin-sensitive tumor. ${ }^{18}$

\section{Stomach}

Studies achieved on Mesenchymal Stromal Cells associated with Gastric Cancer (GC-MSC) have shown how curcumin reduces the angiogenic potential and the metastatic ability in these cells.

The high expression of fibroblastic proteins, such as $\alpha$-SMA and vimentin, in GC-MSC cells leads to the ability to promote tumoral growth, stimulating tumoral cells proliferation, inducing the epithelium-mesenchymal transition in tumoral cells, supporting the expansion of tumoral cells and promoting angiogenesis and metastasis development. Curcumin treatment on GC-MSC cells has blunted the previously mentioned mechanism, reducing the protein expression. ${ }^{3}$

Studies have also examined curcumin's effect towards tumoral angiogenesis, since tumor growth specifically requires the development of new blood vessels, in order to bring additional nutrition and oxygen. It has been noticed that curcumin administration to GC-MSC cells inhibits their pro-angiogenic role, through the inhibition of both endothelial growth factors pathway (VEGF) and NFkB pathway. ${ }^{3}$

Gastric cancer cells show overexpression of COX-2 and abnormal activation of the NF-kB signaling pathway compared to normal gastric mucosa. This suggests that COX-2 and NF-kB inhibitors can be successful in treating gastric cancer. One of the assumptions that have been made is that curcumin could enhance gastric tumor's sensitivity towards chemotherapeutical agents, through the inhibition of NF-kB expression. Studies have shown that the association between 5-fluorouracil (5-FU, used to treat several tumors) and curcumin reduces in a dose-related way the expression of COX-2 and NF-kB. ${ }^{5}$

\section{Lung}

Referring to Non-Small Cells Lung Cancer (NSCLC), studies have shown how curcumin is able to reduce cell growth and colonies development in NSCLC human cells.

In NSCLC, human cells that have received a $10 \mu \mathrm{M}$ dose of curcumin, vitality has been reduced in parallel with apoptosis enhancement. Furthermore, curcumin has been proved to be suc- 
cessful in increasing autophagy mechanisms in lung cancer cells. It has to be recalled that this mechanism can stimulate degradation of cytoplasmic content inside of the cellular lysosomal compartment and that maintaining a constant level of autophagy is a possible therapeutic target for anti-cancer therapy. ${ }^{19,20}$

These results have been obtained through the suppression, made by curcumin, of mTOR/PI3K/AKT signaling pathway, linked to both autophagy and apoptosis processes. ${ }^{19}$

In addition, other studies have shown how curcumin inhibits cellular survival and proliferation, reducing the expression of Axl tyrosine kinase receptor and affecting Axl activation in NCLSC, leading as a result to lung cancer cells apoptosis. In particular, seems that the administration on curcumin in NCLSC cells inhibits Axl phosphorylation, bonding his ligand Gas $6 .{ }^{21}$

Curcumin has proved anti-proliferation effects also on H460 lung cancer cells, and on their chemoresistant versions H460/CisR and H460/TR. The anti-proliferative effect is given by the induction of p21, a molecule that inhibits cyclin-dependent kinase, and the reduction of X-linked Inhibitor of Apoptosis Protein (XIAP), that has an anti-apoptotic action. ${ }^{21}$

\section{Glioblastoma}

Glioblastoma (GBM), for its high proliferation rate and invasive nature, is a low survival rate tumor. Therefore, new molecules are sought in order to enhance the therapeutic performance: curcumin is included between these new molecules, in association with other therapeutic substances.

Firstly, curcumin has been associated with micro RNA (miRNA, small non-coding RNA that regulate genic expression in a post-transcriptional level).

Studies made on animal models and cellular lines show that the association of $50 \mu \mathrm{M}$ of curcumin with miR-378 (miRNA suppressed in GMB cells, that plays a suppressive role on tumors and inhibits tumoral migration and invasion), reduces tumor cells proliferation. ${ }^{22}$

A second curcumin association for GMB treatment is with Nimustine hydrochloride (ACNU), a chemotherapeutic drug commonly used for GMB treatment.

The association of $20 \mu \mathrm{M}$ of curcumin with ACNU has inhibited cellular vitality in GMB cellular cultures. This action seems to be linked to curcumin ability to strengthen ACNU effects, towards the simultaneous action of the cytochrome/caspase-linked apoptotic pathway, the inactivation of PI3K/AKT signal and the inhibition of NF- $\mathrm{KB} / \mathrm{COX}-2$ pathway. ${ }^{23}$

Curcumin and ACNU association seems to be relevant also in chronic inflammation, which may lead to an increment of cancer risk, including malignant brain tumors. A key factor in inflammatory processes is COX-2, associated with cancerogenesis and apoptosis resistance, and whose overexpression is relevant in glioma development. The activation of NF- $\mathrm{B}$ p65/p50 pathway plays a role in COX-2 overexpression.

It has emerged that the association curcumin-ACNU improves the inhibition of COX-2 expression, presumably through repression of NF- $\mathrm{KB}$ dimers translocation from cytosol to nucleus, by canceling COX-2 transcriptional activation in GBM cells. ${ }^{23}$ In addition, the abovementioned association reduces the migration, invasion and metastasization ability of GBM cells towards the down-regulation of MMP-2/9, N-cadherin and vimentin, proteins involved in remote metastasis creation. ${ }^{23}$

\section{Other tumors}

Curcumin had shown its effectiveness also towards other categories of tumors. In particular, studies have proved that: i)
Curcumin inhibits cells proliferation and promotes apoptosis in laryngeal tumor cells, towards a mechanism that entails the alteration of Bcl-2's target miRNA regulation and of PI3K/Akt signaling pathway. Laryngeal tumor cells AMC-HN-8 treated with 40 $\mu \mathrm{M}$ of curcumin has ve shown an enhancement of the apoptotic rate towards the expression of PI3K protein and p-Akt protein $;^{8}$ ii) In pancreatic, hepatic ${ }^{20}$ and prostatic ${ }^{3}$ cells curcumin has diminished the clonogenic capability, inhibited invasion and migration, suppressed cell growth and induced apoptosis and cell cycle arrest. In these cells treated with curcumin, it has been shown an increment in the expression of the proapoptotic molecule Bax, together with the reduction of the anti-apoptotic molecule Bcl-2. Curcumin's inhibitory effect has been higher when administrated at a concentration of $20 \mu \mathrm{M} ;{ }^{20}$ iii) In MCF-7 breast cancer cells, treated with increasing doses of curcumin $(2.5,5,10,20$ and 40 $\mu \mathrm{M})$ it has been observed a reduction of cells proliferation in a dose-related way. When treated with $20 \mu \mathrm{M}$ of curcumin for 24,48 and 72 hours, MCF-7 cells have shown an increment in apoptotic rate, of the amount of pro-apoptotic protein Bax and a reduction of anti-apoptotic protein Bcl-2, proportionally to the amount of time they were kept in culture. ${ }^{20}$

\section{Curcumin role as a therapeutical adjuvant}

Apart from being an antitumoral factor, curcumin, thanks to its negligible toxicity, can be used as an adjuvant in currently in chemotherapies that are already being used for several tumors' treatments.

Curcumin role in such case is to enhance treatment's effects and to improve cellular resistance to drugs. The association between fluorouracil (5-FU) and $20 \mu \mathrm{M}$ of curcumin has proved to be effective in enhancing drug's cytotoxicity in colon cancer cell lines HCT116 and HT29.

The mechanism by which this activity is carried out is the suppression of Akt signaling and autophagic activity, through the reduction of AMPK / ULK1 signaling. ${ }^{24}$ Curcumin has shown to be effective in enhancing the cytotoxic effects of 5-FU also in cell lines of bladder tumor epithelial cells (EJ138).

EJ138 cells, treated with $15 \mu \mathrm{M}$ of curcumin, have shown an increased response to 5-FU, with a markedly increased drug's cytotoxicity, a reduction of cell viability has in addition been observed.

Surprisingly the opposite effect has been observed if cells were treated with 5 -FU and $5 \mu \mathrm{M}$ of curcumin, suggesting that curcumin effects are dose-related. ${ }^{7}$

The therapeutic activity of curcumin has eventually been proved in non-tumoral pathologies such as IBD and periodontal diseases. ${ }^{6}$

In experimental colitis, induced by trinitrobenzene sulfonic acid, curcumin has proved to be effective in reducing the inflammatory effect, towards the inhibition of TLR4, and this suggests that this effect could be mirrored in IBD affected patients. ${ }^{6}$ In periodontal diseases (chronic inflammatory diseases caused by an immune response towards bacteria located in dental biofilm), curcumin reduces the innate immune responses, by modulating TLR signal. ${ }^{6}$

\section{Conclusions}

A significant number of studies show curcumin's antitumoral potential on different kinds of tumors, suggesting that that results obtained during the in-vitro studies, could potentially be found also in in-vivo studies. These results, if confirmed in future studies on 
human beings, could have a strong impact on therapeutic choices, especially considering the high incidence of pathologies such as colorectal cancer, or such as the ones whose main risk is represented by intestinal polyp's progression towards malignancy. Remarkable is the contribution that could result from curcumin usage in those tumors characterized by a lower incidence but with a decreased rate of survival, such as pancreas tumor and glioblastoma. Curcumin role in pro-apoptotic and anti-angiogenic processes, found in Non-Small Cells Lungs Cancer (NSCLC) and gastric cancer, has to be used as an input for further studies. Nevertheless, it has to be pointed out that the results obtained and the subsequent assumptions of the application on human beings are based on murine models or cell culture. To this day there are neither evidence nor studies that could confirm the antitumoral and therapeutic potential of curcumin on human beings. In addition, there are no strong pieces of evidence that, in vivo, the association of curcumin and chemotherapy drugs improve prognosis and survival rate in patients affected by tumors.

\section{References}

1. Zhu Y, Bu S. Curcumin induces autophagy, apoptosis, and cell cycle arrest in human pancreatic cancer cells. Evid Based Complement Alternat Med 2017;5787218.

2. Murray-Stewart T, Casero RA. Regulation of polyamine metabolism by curcumin for cancer prevention and therapy. Med Sci 2017;18;5.

3. Huang F, Yao Y, Wu J, et al. Curcumin inhibits gastric cancerderived mesenchymal stem cells mediated angiogenesis by regulating NF- $\mathrm{B} / \mathrm{VEGF}$ signaling. Am J Transl Res 2017;9:5538-47.

4. Cruz-Correa M, Shoskes DA, Sanchez P, et al. Combination treatment with curcumin and quercetin of adenomas in familial adenomatous polyposis. Clin Gastroenterol Hepatol 2006;4: 1035-8.

5. Yang H, Huang S, Wei Y, et al. Curcumin Enhances the Anticancer Effect Of 5-fluorouracil against Gastric Cancer through Down-Regulation of COX-2 and NF- $\kappa$ B Signaling Pathways. J Cancer 2017;8:3697-706.

6. Boozari M, Butler AE, Sahebkar A. Impact of curcumin on toll-like receptors. J Cell Physiol 2019;234:12471-82.

7. Afsharmoghadam N, Haghighatian Z, Mazdak H, et al. Concentration- Dependent Effects of Curcumin on 5Fluorouracil Efficacy in Bladder Cancer Cells. Asian Pac J Cancer Prev 2017;18:3225-30.

8. Mou S, Zhou Z, He Y, et al. Curcumin inhibits cell proliferation and promotes apoptosis of laryngeal cancer cells through Bcl-2 and PI3K/Akt, and by upregulating miR-15a. Oncol Lett 2017;14:4937-42.

9 . Mortezaee K, Salehi E, Mirtavoos-Mahyari H, et al. Mechanisms of apoptosis modulation by curcumin: Implications for cancer therapy. J Cell Physiol 2019;234:12537-50.

10. Carini F, David S, Tomasello G, et al. Colorectal cancer: an update on the effects of lycopene on tumor progression and cell proliferation. J Biol Regul Homeost Agents 2017;31:769-74.

11. Carini F, Mazzola M, Rappa F, et al. Colorectal carcinogenesis: role of oxidative stress and antioxidants. Anticancer Res 2017;37:4759-66.

12. Tomasello G, Mazzola M, Leone A, et al. Nutrition, oxidative stress and intestinal dysbiosis: Influence of diet on gut microbiota in inflammatory bowel diseases. Biomed Pap Med Fac Univ Palacky Olomouc Czech Repub 2016;160:461-6.

13. Mazzola M, Carini F, Leone A, et al. Inflammatory bowel disease and colorectal cancer, nutraceutical aspects. Euromediterranean Biomed J 2016;11:123-9.

14. Carini F, Tomasello G, Jurjus A, et al. Colorectal cancer and inflammatory bowel diseases: effects of diet and antioxidants. J Biol Regul Homeost Agents 2017;31:791-5.

15. Mazzola M, Carini F, Leone A, et al. Ibd, malignancy and oral microbiota: analysis of the literature. Int J Clin Dent 2016;9: 273-8.

16. Alfonso-Moreno V, López-Serrano A, Moreno-Osset E. Chemoprevention of polyp recurrence with curcumin followed by silibinin in a case of multiple colorectal adenomas. Rev Esp Enferm Dig 2017;109:875.

17. Dou H, Shen R, Tao J, et al. Curcumin suppresses the colon cancer proliferation by inhibiting Wnt/ $\beta$-Catenin pathways via miR-130a. Front Pharmacol 2017;8:877.

18. Dhillon N, Aggarwal BB, Newman RA, et al. Phase II trial of curcumin in patients with advanced pancreatic cancer. Clin Cancer Res 2008;14:4491-9.

19. Wang A, Wang J, Zhang S, et al. Curcumin inhibits the development of non-small cell lung cancer by inhibiting autophagy and apoptosis. Exp Ther Med 2017;14:5075-80.

20. Liu JL, Pan YY, Chen O, et al. Curcumin inhibits MCF-7 cells by modulating the NF- $\kappa \mathrm{B}$ signaling pathway. Oncol Lett 2017;14:5581-4.

21. Kim KC, Baek SH, Lee C. Curcumin-induced downregulation of Axl receptor tyrosine kinase inhibits cell proliferation and circumvents chemoresistance in non-small lung cancer cells. Int J Oncol 2015;47:2296-303.

22. Li W, Yang W, Liu Y, et al. MicroRNA-378 enhances inhibitory effect of curcumin on glioblastoma. Oncotarget 2017;8: 73938-46.

23. Zhao J, Zhu J, Lv X, et al. Curcumin potentiates the potent antitumor activity of ACNU against glioblastoma by suppressing the PI3K/AKT and NF- $\mathrm{KB} / \mathrm{COX}-2$ signaling pathways. Onco Targets Ther 2017;10:5471-82.

24. Zhang P, Lai ZL, Chen HF, et al. Curcumin synergizes with 5fluorouracil by impairing AMPK/ULK1-dependent autophagy, AKT activity and enhancing apoptosis in colon cancer cells with tumor growth inhibition in xenograft mice. J Exp Clin Cancer Res 2017;36:190. 